\title{
Task-irrelevant auditory feedback facilitates motor performance in musicians
}

\author{
Virginia Conde ${ }^{1}$, Eckart Altenmüller ${ }^{2}$, Arno Villringer ${ }^{1,3}$ and Patrick Ragert ${ }^{1}{ }^{*}$ \\ ${ }^{1}$ Department of Cognitive Neurology, Max Planck Institute for Human Cognitive and Brain Sciences, Leipzig, Germany \\ 2 Institute for Music Physiology and Musicians Medicine, Hanover, Germany \\ ${ }^{3}$ Mind Brain Institute, Charité and Humboldt University, Berlin, Germany
}

\section{Edited by:}

Angela Dorkas Friederici, Max Planck Institute for Human Cognitive and

Brain Sciences, Germany

Reviewed by:

Lutz Jäncke, University of Zurich, Switzerland

Barbara Tillmann, Centre national de la recherche scientifique, France

\section{*Correspondence:}

Patrick Ragert, Department of Cognitive Neurology, Max Planck Institute for Human Cognitive and Brain Sciences, Stephanstrasse 1a, D-04103 Leipzig, Germany. e-mail: ragert@cbs.mpg.de
An efficient and fast auditory-motor network is a basic resource for trained musicians due to the importance of motor anticipation of sound production in musical performance. When playing an instrument, motor performance always goes along with the production of sounds and the integration between both modalities plays an essential role in the course of musical training. The aim of the present study was to investigate the role of task-irrelevant auditory feedback during motor performance in musicians using a serial reaction time task (SRTT). Our hypothesis was that musicians, due to their extensive auditory-motor practice routine during musical training, have superior performance and learning capabilities when receiving auditory feedback during SRTT relative to musicians performing the SRTT without any auditory feedback. Behaviorally, we found that auditory feedback reinforced SRTT performance of the right hand (referring to absolute response speed) while learning capabilities remained unchanged. This finding highlights a potential important role for task-irrelevant auditory feedback in motor performance in musicians, a finding that might provide further insight into auditory-motor integration independent of the trained musical context.

Keywords: audio-motor integration, motor learning, musicians, serial reaction time task, auditory feedback

\section{INTRODUCTION}

Auditory-motor integration plays an essential role in a variety of multisensory tasks, becoming indispensable for the performance of music. When playing an instrument, the interaction between the auditory and motor cortex seems to be an essential prerequisite since every motor action produces a corresponding sound (Zatorre et al., 2007). For professional musicians it has been hypothesized that an efficient and fast auditory-motor network is a basic resource that presumably gets reinforced by practice (Schulz et al., 2003; Baumann et al., 2007; Hyde et al., 2009; Pantev et al., 2009).

Auditory-motor integration in the context of musical practice has been previously examined with different experimental designs. For example, Pantev et al. (2009) showed a greater enhancement of musical representations in the auditory cortex after a sensorimotor-auditory training as compared to sole auditory training. In a similar fashion, motor cortex excitability has been shown to increase during a mere auditory presentation of a rehearsed musical piece (sensorimotor-auditory coupling). Interestingly, no such changes were observed for an auditory presentation of a non-rehearsed musical piece (D'Ausilio et al., 2006).

It has been suggested that professional musicians have stronger connections between auditory and motor areas relative to nonmusicians, resulting in a superior translation of finger actions into auditory music representations (Baumann et al., 2007). This stronger connection in turn might lead to an increased plasticity in both auditory and motor cortices as a type of cross-modal plasticity (Lim et al., 2005). However, at a more basic level, little is known about the role of irrelevant auditory feedback over the motor cortex in musicians independently of a specific musical context.

The aim of the present study was to investigate the role of taskirrelevant auditory feedback in musicians during visually guided motor performance using a serial reaction time task (SRTT). In a similar manner as previously done by Stocker et al. (2003), we designed two different SRTTs that required motor performance guided by visual cues. In our study, the only difference between both SRTTs was the presentation of either task-irrelevant or no auditory feedback. By using a response-button device (RBD) instead of a piano keyboard, we aimed to disentangle the role of auditory feedback over motor performance under a more general framework in musicians. The SRTT was designed not to be directly connected to music performance when playing a specific instrument or to an extensively trained and therefore well-known auditory-motor map.

In comparison to other studies investigating cross-modal interactions in musicians, we used task-irrelevant auditory feedback to show that auditory-motor integration is not exclusively shaped by context specific multisensory tasks such as playing an instrument. Due to the repeated exposure of musicians to auditory-motor multisensory integration during music skill practice, we hypothesized that SRTT motor performance will be facilitated by taskirrelevant auditory feedback in comparison to SRTT performance without any auditory feedback. 


\section{MATERIALS AND METHODS PARTICIPANTS}

The study was carried out in a total number of 24 healthy highly trained musicians ( 1 out of 24 left-handed; mean age $24.38 \pm 3.47$ years, 11 males). They gave written informed consent to participate in the experiment according to the declaration of Helsinki and the local ethic committee of the University of Leipzig approved the study. Handedness was assessed by the Edinburgh inventory scale (Oldfield, 1971; laterality score: $87.19 \pm 15.30$ and -84.6 for the left-handed musician, over a range of -100 (fully left-handed) and +100 (fully right-handed) where a value of 0 was used as a cut-off score between right and left handers). Musicians were recruited at the Institute of Music Physiology and Musicians Medicine in Hannover; all of them were experienced piano players as first or second instrument with an average starting age of $6.83 \pm 2.48$ and $12.15 \pm 5.78$ years for other instruments (for details, see Table 1). The present average time for piano playing was $2.52 \pm 1.78 \mathrm{~h}$ per day and $2.13 \pm 1.28 \mathrm{~h}$ per day in average lifetime. Six of the musicians reported possessing absolute pitch.

\section{STUDY DESIGN}

In the present study, we used a SRTT that did not imply the use of any musical instrument. The SRTT consisted of sequential motor responses to a series of visual stimuli displayed on a computer screen using a customized script written with Presentation software (Neurobehavioral systems, Inc., Albany, CA, USA). Participants seated in front of the computer screen with both hands placed on a table where a RBD was attached. The RBD had four buttons ergonomically oriented with respect to the right hand (see Figure 1). We decided to use a mixed key-tone mapping for the RBD that did not keep left-to-right organization of pitch in a real piano keyboard in order to avoid the activation of already existent

Table 1 | Musical biography of musicians $(n=24)$ performing the SRTT (age, first instrument, second instrument, starting ages for both first and second instruments, average lifetime hours of practice per day, average at present hours of practice per day).

\begin{tabular}{|c|c|c|c|c|c|c|c|}
\hline Subject & Age & First instrument & Start age (years) & Second instrument & $\begin{array}{l}\text { Start age } \\
\text { (years) }\end{array}$ & $\begin{array}{l}\text { Average lifetime } \\
\text { (h/day) }\end{array}$ & $\begin{array}{l}\text { At present } \\
\text { (h/day) }\end{array}$ \\
\hline 01 & 24 & Piano & 13 & Drums & 17 & 1.83 & 1 \\
\hline 03 & 20 & Piano & 7 & Guitar & 15 & 0.53 & 1 \\
\hline 04 & 23 & Piano & 7 & Harpsichord/Pianoforte & 21 & 4.25 & 4 \\
\hline 05 & 24 & Piano & 6 & Drums & 12 & 0.75 & 1 \\
\hline 07 & 26 & Piano & 5 & Cello & 10 & 2.00 & 3 \\
\hline 08 & 22 & Violin & 5 & Piano & 8 & 1.44 & 2 \\
\hline 09 & 20 & Piano & 4 & Violin & 5 & 1.17 & 3 \\
\hline 10 & 24 & Piano & 8 & Guitar & 14 & 1.63 & 1 \\
\hline 11 & 21 & Piano & 5 & Transverse/flute & 15 & 1.94 & 1.5 \\
\hline 16 & 26 & Piano & 3 & Harpsichord & 5 & 2.50 & 4 \\
\hline 17 & 23 & Piano & 7 & - & - & 3.94 & 5.5 \\
\hline 18 & 28 & Piano & 4 & - & - & 2.00 & 5.5 \\
\hline 19 & 29 & Piano & 7 & Viola & 21 & 1.40 & 1 \\
\hline 20 & 26 & Piano & 6 & Saxophone & 21 & 1.63 & 1 \\
\hline 21 & 27 & Piano & 5 & - & - & 5.30 & 6 \\
\hline 22 & 27 & Piano & 7 & Viola & 7 & 4.20 & 5 \\
\hline 23 & 26 & Trumpet & 7 & Piano & 6 & 1.50 & 1.25 \\
\hline 24 & 31 & Violoncello & 6 & Piano & 12 & 0.56 & 0.25 \\
\hline \multicolumn{3}{|l|}{ Factors } & \multicolumn{2}{|l|}{ VM } & \multicolumn{2}{|l|}{ VMA } & $p$ Value \\
\hline
\end{tabular}

Dark gray shading indicates subjects that were allocated to the VM group. Summary of group data and group comparisons (independent samples t-tests) for factors "age," "starting age (piano)," "lifetime piano practice in hours per day," and "at present piano practice in hours per day." All data are expressed as average group values \pm standard deviation. 
A

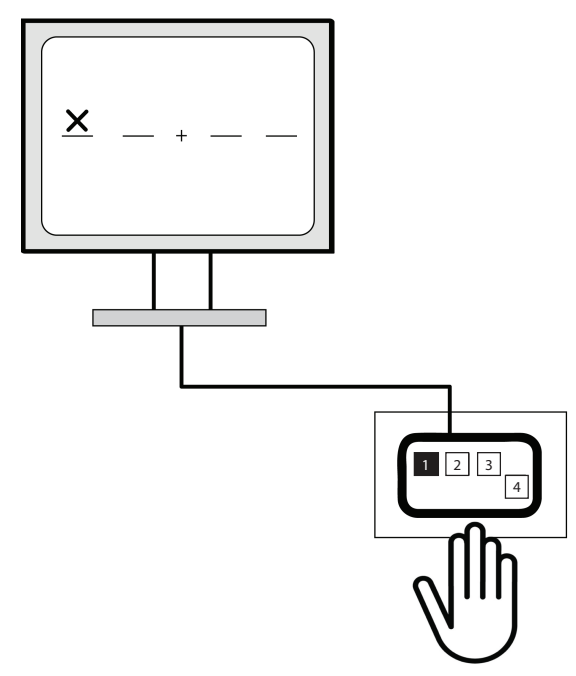

B

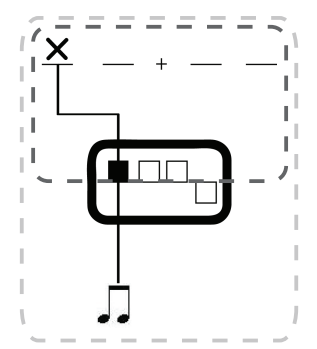

C

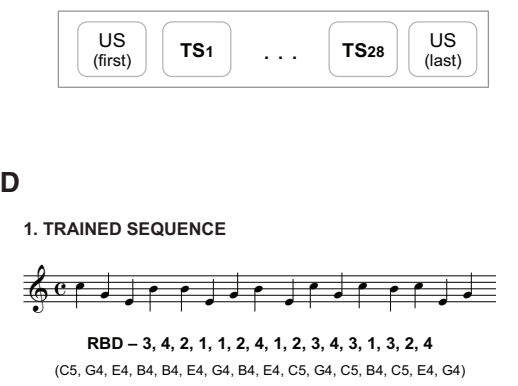

2. UNTRAINED SEQUENCE

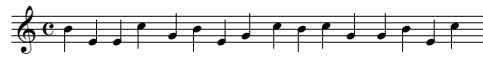

RBD - 1, 2, 2, 3, 4, 1, 2, 4, 3, 1, 3, 4, 4, 1, 2, 3 $\mathrm{B} 4, \mathrm{E} 4, \mathrm{E} 4, \mathrm{C} 5, \mathrm{G} 4, \mathrm{~B} 4, \mathrm{E} 4, \mathrm{G} 4, \mathrm{C} 5, \mathrm{~B} 4, \mathrm{C5}, \mathrm{G} 4, \mathrm{G} 4, \mathrm{~B} 4, \mathrm{E} 4 ; \mathrm{C} 5)$
FIGURE 1 | (A) Schematic representation of the serial reaction time task (SRTT). The SRTT consisted of sequential motor responses to a series of visual stimuli $(X)$ displayed on a computer screen. Participants had to respond as fast as possible when the visual stimuli appeared on the screen with a respective button press on a response box (illustrates as the black square for example). The only difference between the SRTT conditions (B) was that in the visuo-motor-auditory condition, participants hear an auditory signal each time they pressed a button (dashed light gray box) while in the visuo-motor condition (dashed dark gray box) participants did not receive any auditory feedback after button pressing. For details see text. (C) At the beginning of the SRTT, participants had to perform a untrained sequence [US(first)] followed by 28 repetitions of a trained sequence (TS1-TS28). After TS28, the untrained sequence was repeated again [US(last)]. (D) Sequence order for the trained (TS) and untrained sequence (US) and corresponding tones. 1, 2, 3, and 4 corresponds to a button press with the right index-, middle-, ring-, and little-finger in the response-button device (RBD), respectively. motor-auditory representations. The visual presentation on the computer screen consisted of four horizontal lines with a fixation cross in the middle where an " $X$ " appeared changing positions along each horizontal line with a constant interstimulus interval (ISI) of $900 \mathrm{~ms}$. The fixed ISI was used as a pacemaker and the subjects were instructed to keep the tempo of such ISI as a visual metronome. This experimental setup was used to make the task more "musical-like" without the use of a classical piano keyboard. Each button on the device corresponded to a specific line of the visual presentation and the " $\mathrm{X}$ " served as a visual cue (according to previous SRTT tasks; Perez et al., 2008) indicating which key to press (see Figure 1). During the SRTT, participants learned a specific sequence that was presented 28 times (trained sequence; TS1 to TS28). Additionally, one untrained sequence (US) was presented before and after the trained sequence trials. Each sequence contained 16 movements of the " $\mathrm{X}$ " across the four horizontal lines and there were 30 trials in total. Each sequence started with the same event (see also Figure 1). A similar approach was used in other SRTT tasks before (Perez et al., 2007, 2008). Participants were asked to respond as fast as possible once they perceived the "X" by pressing the corresponding button on the RBD.

Reaction times (RTs) were measured and defined as the time interval between the GO signal ("X") and the correct key press.

All participants were task naive and were not aware about a specific sequence order. Feedback regarding average RT and number of errors was given by the end of each trial. There were two possible SRTT conditions depending on the presence of auditory feedback: visuo-motor (VM) and visuo-motor-auditory (VMA) condition. Participants were randomly assigned to one of the two conditions, resulting in two different groups of participants (musicians VM, 12 participants; musicians VMA, 12 participants). Headphones were used for all conditions and participants were asked to use them for better sound isolation. In the VMA condition, sampled piano tones (Apple Logic, software instrument "Steinway Hall") from a C major scale (B4, button 1; E4, button 2; C5, button 3; G4, button 4 ; with only one misplaced tone to the $\mathrm{C}$ scale) with a duration of $200 \mathrm{~ms}$ were generated simultaneously with each motor response, while each button of the RBD always generated the same tone. Tones were ordered for US (B4, E4, E4, C5, G4, B4, E4, G4, C5, B4, C5, G4, G4, B4, E4, C5) and TS (C5, G4, E4, B4, B4, E4, G4, B4, E4, C5, G4, C5, B4, C5, E4, G4) without taking into account melodic or harmonic features (see Figure 1D; for an audio file of each sequence please see Supplementary Material). Participants in the VMA condition were instructed about the auditory feedback as non-related with the task performance. Participants were asked to fill out a questionnaire concerning musical expertise before the SRTT started. Additionally, all participants rated their individual level of fatigue and discomfort using a visual analog scale (VAS) ranging from 1 to 10 ( 1 meaning very tired/very uncomfortable) before and after the SRTT. RT in ms, expressed as time lag between the visual cue and corresponding button press, was recorded for offline analysis.

\section{BEHAVIORAL MEASUREMENTS}

Different aspects of the SRTT were measured such as performance, performance improvement, learning, and error rates. Performance was defined as raw average RT for each trial considering correct responses only, while performance improvement was considered as 
the difference between the last TS and the first TS (TS1-TS28). In order to define the individual learning effect in the SRTT we used the following formula [(TS1-TS28)/(US(first)-US(last))], which takes into account both trained and USs to rule out unspecific learning effects due to repeated button presses. Additionally, we computed the difference from the last (second) US to the last TS [US(last)-TS28] in order to get another focal measure of learning considering the last trained sequence vs. the last US only. Finally, error was defined as incorrect key press relative to the " $\mathrm{X}$ " position upon the horizontal lines.

\section{STATISTICAL ANALYSIS}

Data analysis was performed using PASW for Windows version 18. In order to compare the musical biography of the musicians for each condition (VM and VMA) we used one-way ANOVAs with factors "starting age piano training," "piano training lifetime," and "piano training at present." The statistical analysis was divided into performance, performance improvement, learning, and error rates as described above. For performance, a repeated measures ANOVA (ANOVARM) was applied for each condition (withinsubjects analyses; VM, and VMA), as well as a one-way ANOVA with factor CONDITION (between-subjects analysis; VM, VMA) to compare "average RTs" of the 28 trials in the TS between conditions. Furthermore, we used an ANOVARM in order to compare the effect of time (factor TIME; 28 repeated measures of the TS) relative to condition (VM, VMA), for which a TIME x CONDITION interaction was tested. For the comparison of learning effect and error rates between conditions, one-way ANOVAs (factor CONDITION; VM, VMA) were applied. One-sample Student's $t$-tests were applied in order to determine the significance of the sequence-specific (TS) and unspecific (US) learning effect within condition, for which we analyzed the percentage of the difference between US(first) and US(last) to have a measure of RT changes not related with the repeated sequence (TS) as well as the percentage change of the difference between TS1 and TS28. Finally, we performed Pearson correlations among behavioral measurements (SRTT performance improvement, learning, and average RT) and musical expertise (starting age, at present hours of practice per day and lifetime hours of practice per day). Multiple comparisons correction was performed using Šidàk correction for nine comparisons (nine correlations; $p=0.006$ ).

\section{RESULTS}

Comparing the VAS before (pre) and after (post) the behavioral experiment revealed no significant change in fatigue and discomfort levels between conditions (VM and VMA; $p>0.05$ in all cases) and within participants (pre and post; $p>0.05$ in both conditions, please, see also Table 2 for details).

\section{MUSICAL BIOGRAPHY}

There were no significant differences between the VM and VMA group with respect to starting age of piano practice, average lifetime hours of training and average hours of training at present as analyzed with an one-way ANOVA [starting age: $F(1,22)=0.26$, $p=0.873$; average lifetime hours of training: $F(1,22)=1.64$, $p=0.213$; average hours of training at present: $F(1,22)=1.11$, $p=0.302$; see Table 1 ].

\section{SRTT PERFORMANCE SRTT VM}

Performing the SRTT resulted in a significant difference between US(first) and US(last; $p=0.005$ ) with average RTs of $387.50 \pm 16.39 \mathrm{~ms}$ [US(first)] and $332.33 \pm 8.63 \mathrm{~ms}$ [US(last)]. The performance in US(last) was $12.80 \pm 3.65 \%$ faster than US(first) indicating an unspecific performance improvement in the SRTT (see Figure 3). ANOVARM with factor TIME showed a significant procedural improvement along trials in TS [Mauchly's test of sphericity: $p>0.05 ; F(27,297)=17.899 ; p<0.001]$. The average RT in TS28 was $37.19 \pm 5.01 \%$ faster as compared to TS1. Comparing TS vs. US SRTT performance improvements revealed that improvements in TS were significantly higher as compared to US $(p<0.001)$.

\section{SRTT VMA}

As in the SRTT VM condition, SRTT performance in VMA resulted in a significant difference between US(first) and US(last; $p=0.001$ ) with average RTs of $330.50 \pm 10.42 \mathrm{~ms}$ [US(first)] and $282.67 \pm 8.10 \mathrm{~ms}$ [US(last)]. The performance on the US(last) was $13.86 \pm 3.02 \%$ faster than US(first) indicating an unspecific SRTT improvement (see Figure 3). With respect to the TS specific improvement, there was a significant difference between TS1 and TS28 (average RTs: $301.25 \pm 8.22 \mathrm{~ms}$ for TS1, $210.50 \pm 11.09 \mathrm{~ms}$ for TS28), with TS28 being $28.28 \pm 4.59 \%$ faster. The improvement in TS was significantly higher than the improvement in US $(p=0.027)$.

ANOVARM with factor TIME revealed a significant TS performance improvement along trials [Mauchly's test of sphericity: $p>0.05 ; F(27,297)=14.500 ; p<0.001]$.

\section{COMPARISON BETWEEN SRTT VM AND VMA}

We found a significant difference between SRTT VM and VMA. Musicians in the VMA condition had a significantly faster performance (average $\mathrm{RT}=249.71 \pm 4.94 \mathrm{~ms}$ ) than musicians in the VM condition (average RT $=284.63 \pm 8.74 \mathrm{~ms}$; see Figure 2B) when considering overall average RT in the TS [one-way ANOVA factor CONDITION; $F_{(1,23)}=12.089, p=0.002$ ] (see Figure 2B). Interestingly, when addressing both $\mathrm{VM}$ and VMA performance along time (considering all trials of the TS) with an ANOVARM factor TIME x CONDITION, we found a significant interaction between both factors [Mauchly's test of sphericity: $p>0.05 ; F(27,297)=2.149 ; p=0.001]$, indicating that the time course of improvement resulted in a different performance curve for each condition (VM, VMA; see Figure 2A). Similar results were obtained when comparing RT improvements (TS1TS28) between conditions [one-way ANOVA factor CONDITION; $\left.F_{(1,23)}=4.920, p=0.037\right]$. Musicians in the VM condition showed larger RT improvements within TS as compared to musicians in the VMA condition (average RT improvement: $147.58 \pm 22.00 \mathrm{~ms}$ in VM; $90.75 \pm 13.14$ ms; see Figure 2A). In order to exclude that potential differences in RT in the very first trial (first button press) are potentially driving the significant finding mentioned above, an ad hoc RT of the first trial was computed from the first button press in each subject [average RT $(\mathrm{VM})=404 \pm 84.51 \mathrm{~ms}$; average $\mathrm{RT}(\mathrm{VMA})=362 \pm 98.18 \mathrm{~ms}]$. There was, however, no significant difference between groups $(p=0.273)$. 
Table 2 | Levels of fatigue (1 = strong fatigue, $10=$ no fatigue), discomfort (1 = strong discomfort, $10=$ no discomfort) before (pre) and after (post) the experiment as well as error rate across the SRTT (wrong key presses for all trials tested) for the VM and VMA group.

\begin{tabular}{|c|c|c|c|c|c|}
\hline \multirow[t]{3}{*}{ Subjects } & \multicolumn{4}{|c|}{ VM group } & \multirow[t]{3}{*}{ Error rate } \\
\hline & \multicolumn{2}{|c|}{ Fatigue } & \multicolumn{2}{|c|}{ Discomfort } & \\
\hline & Pre & Post & Pre & Post & \\
\hline VP02 & 9 & 7 & 10 & 8 & 29 \\
\hline VP04 & 2 & 3 & 8 & 7 & 18 \\
\hline VP05 & 7 & 8 & 8 & 8 & 18 \\
\hline VP06 & 7 & 4 & 8 & 4 & 33 \\
\hline VP11 & 10 & 8 & 9 & 8 & 16 \\
\hline VP12 & 2 & 4 & 8 & 9 & 11 \\
\hline VP13 & 6 & 5 & 8 & 7 & 14 \\
\hline VP16 & 9 & 9 & 10 & 10 & 11 \\
\hline VP17 & 7 & 8 & 5 & 5 & 26 \\
\hline VP19 & 7 & 9 & 7 & 6 & 10 \\
\hline VP20 & 6 & 4 & 9 & 8 & 14 \\
\hline VP24 & 3 & 3 & 2 & 3 & 14 \\
\hline Mean & 6.25 & 6.00 & 7.67 & 6.92 & 17.83 \\
\hline $\mathrm{SD}$ & 2.67 & 2.37 & 2.23 & 2.07 & 7.53 \\
\hline Sterr & 0.77 & 0.69 & 0.64 & 0.60 & 2.35 \\
\hline
\end{tabular}

Subjects

VMA group

Error rate

\begin{tabular}{|c|c|c|c|c|c|}
\hline & & \\
\hline & \multicolumn{2}{|c|}{ Fatigue } & \multicolumn{2}{|c|}{ Discomfort } & \\
\hline & Pre & Post & Pre & Post & \\
\hline VP01 & 9 & 8 & 10 & 10 & 41 \\
\hline VP03 & 8 & 7 & 9 & 9 & 22 \\
\hline VP07 & 8 & 9 & 8 & 9 & 4 \\
\hline VP08 & 5 & 5 & 8 & 8 & 10 \\
\hline VP09 & 8 & 4 & 10 & 6 & 10 \\
\hline VP10 & 5 & 8 & 5 & 7 & 17 \\
\hline VP14 & 7 & 9 & 4 & 3 & 25 \\
\hline VP15 & 9 & 10 & 9 & 8 & 22 \\
\hline VP18 & 3 & 7 & 8 & 8 & 15 \\
\hline VP21 & 4 & 4 & 5 & 5 & 31 \\
\hline VP22 & 3 & 4 & 8 & 8 & 25 \\
\hline VP23 & 5 & 3 & 6 & 3 & 49 \\
\hline Mean & 6.17 & 6.50 & 7.50 & 7.00 & 22.58 \\
\hline $\mathrm{SD}$ & 2.25 & 2.39 & 2.02 & 2.30 & 13.04 \\
\hline Sterr & 0.65 & 0.69 & 0.58 & 0.66 & 3.18 \\
\hline$p$-Values & & & & & \\
\hline Across groups & Pre & Post & Pre & Post & \\
\hline (VM, VMA) & 0.934 & 0.612 & 0.850 & 0.926 & 0.286 \\
\hline$p$-Values & Fatigue & & Discomfort & & \\
\hline \multicolumn{6}{|c|}{ WITHIN GROUP (PRE, POST) } \\
\hline VM & 0.622 & & 0.081 & & \\
\hline VMA & 0.608 & & 0.308 & & \\
\hline
\end{tabular}

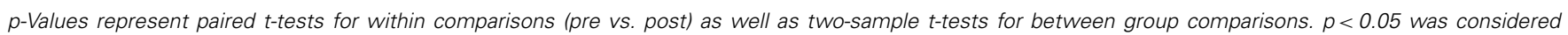
significant.

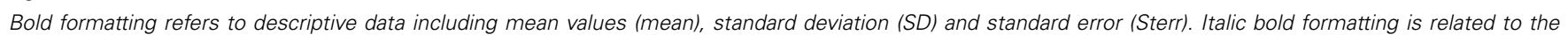
statistical comparsions (paired/two sample t-tests). 
A

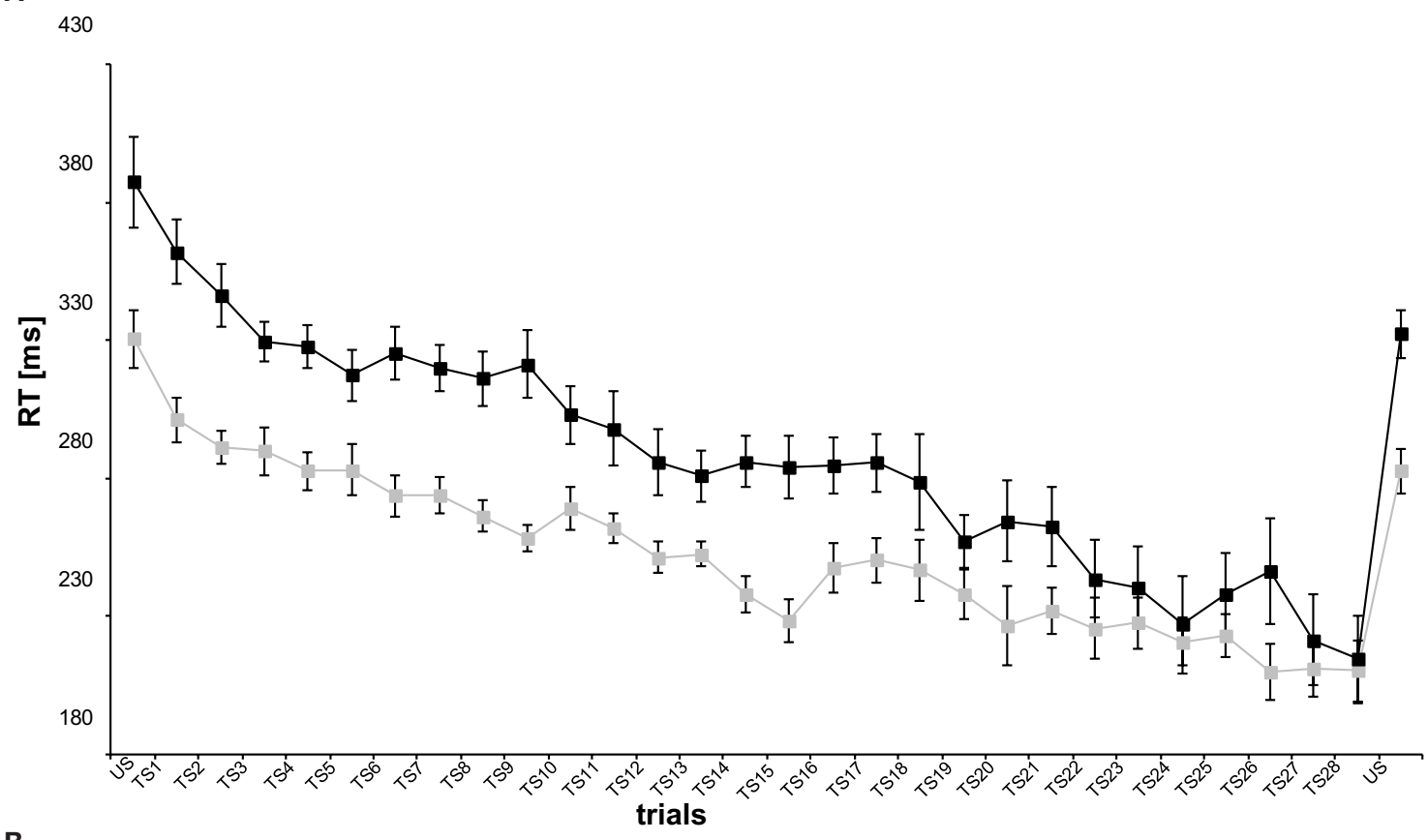

B

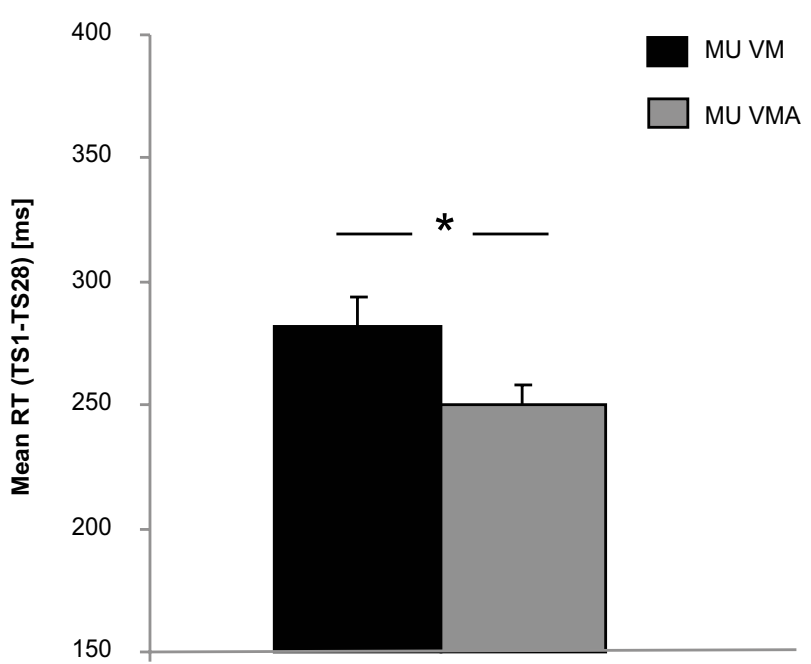

FIGURE 2 | (A) Average reaction times (RT; ms) for the trained sequence (TS) per trial (TS1-28) and the untrained sequence (US) (B) mean RT in SRTT for TS (TS1-28). Bars represent standard errors. Please note that task-irrelevant auditory feedback potentiated motor

\section{SRTT LEARNING}

Musicians in the VM and VMA condition showed a significant learning effect in TS relative to US as indexed by our defined learning parameter [(TS1-TS28)/(US(first)-US(last)) $](p<0.001$ in both conditions). There was a mild tendency for superior learning capabilities of musicians in VM condition as compared to musicians in VMA condition when taking into account both differences between first and last TS and US trials [(TS1-TS28)/(US(first)-US(last))] (percentage of learning: $33.37 \pm 8.11 \%$ in $\mathrm{VM}$ condition; $25.03 \pm 5.05 \%$ in VMA performance in the VMA condition by means of reduced reaction time when compared to the VM group. Legend: MU VM refers to musicians in the VM condition, MUVMA refers to musicians in the VMA condition. condition) and the last US-TS [US(last)-TS28] comparison only (difference in RT (ms): $117.83 \pm 20.51 \mathrm{~ms}$ in VM condition; $72.16 \pm 11.34 \mathrm{~ms}$ in VMA condition) that, nonetheless, did not reach significance [one-way ANOVA factor CONDITION; $F_{(1,23)}=0.675 ; p=0.420 ; F_{(1,23)}=3.796 ; p=0.064$, respectively; see Figures $2 \mathrm{~A}$ and 3 ].

\section{ERROR RATE}

No significant differences were found between conditions in error rate $(p=0.286)$. 


\section{CORRELATION BETWEEN SRTT PERFORMANCE AND STARTING AGE IN MUSICIANS}

In order to find a direct relationship between SRTT performance (VM or VMA) in musicians with their musical expertise or their individual training routine, we performed nine correlation analyses. Among all the correlations performed, there was only a significant negative correlation between starting age and RT improvements (TS1-TS28) in TS VMA condition ( $\mathrm{df}=10$; $\rho=-0.737 ; p=0.006$, see also Figure 4; Table 1), indicating that the earlier a musician started playing the instrument, the larger the improvement in VMA RT was.

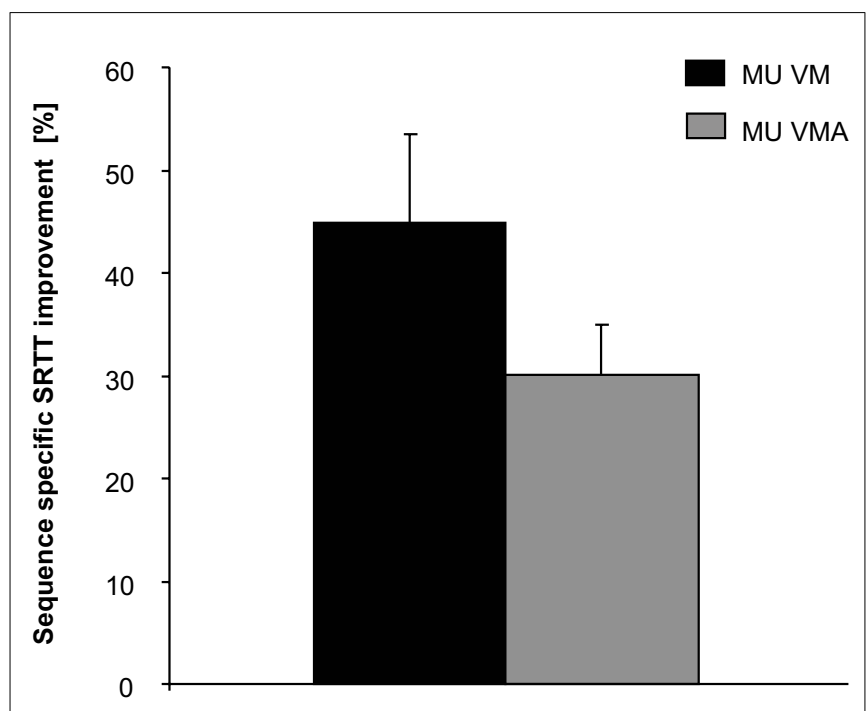

FIGURE 3 | Sequence-specific SRTT improvements (\%). Significant results are symbolized by an asterisk. For details see text. Upper bars represent standard error. Legend: MU VM refers to musicians in the VM condition, MU VMA refers to musicians in the VMA condition.

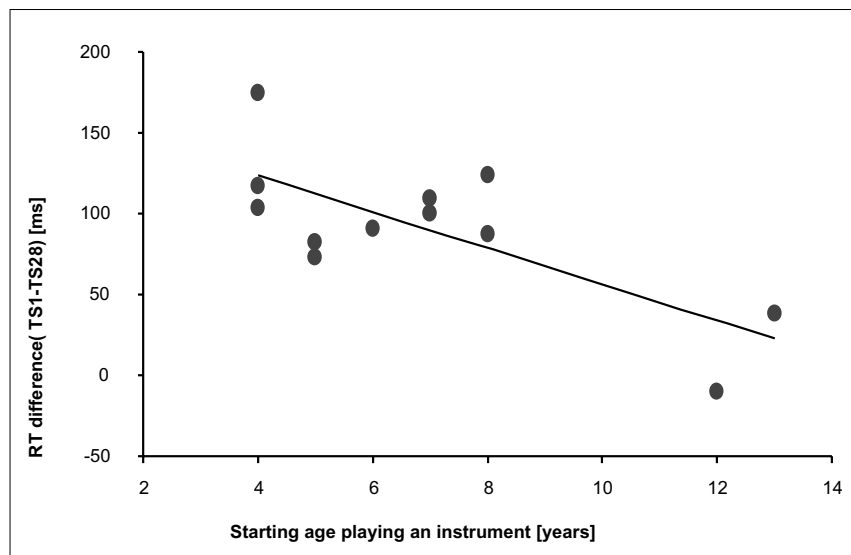

FIGURE 4 | Negative correlation between RT differences [ms] (TS1 vs. TS28) and musicians' starting age for playing an instrument (years). The results indicate that musicians who started playing an instrument early in life were those that showed the largest RT difference between TS1 and TS28 indicating more prominent performance improvements relative to those that started later in life.

\section{DISCUSSION}

In the present study we provided novel evidence that taskirrelevant auditory feedback in a SRTT is associated with better motor performance but not learning in musicians. Musicians who received auditory feedback (VMA) were faster than musicians who just performed the visuo-motor task (VM; see Figures 2A,B). Moreover, a significant correlation between the individual starting age of piano training and the performance improvements of musicians in the VMA condition underscores the importance of use-dependent cross-modal reorganization and its transfer to tasks that share the same modalities of music performance (near transfer, Hyde et al., 2009). The fact that such correlation was only shown in the VMA group could be related to the nature of the task, since the visuo-motor-auditory condition shares more resources with a real musical task than the visuo-motor condition, indicating that the context of the task might be of importance when considering near transfer of skills (Bangert et al., 2006). Nevertheless, our results are in line with a previous study showing a relation between tapping skills and the age of musicians at which they began their musical training (Jancke et al., 1997).

In agreement with our results, there is a huge body of evidence for the existence of a cross-modal functional correlate of this trained auditory-motor integration in musicians in the context of musical tasks (Bangert et al., 2001, 2006; Bangert and Altenmuller, 2003; Lahav et al., 2005; D’Ausilio et al., 2006; Baumann et al., 2007; Pantev et al., 2009). For example, Pantev et al. (2009) showed enhanced plasticity in the auditory cortex after a multimodal sensorimotor-auditory training as compared to auditory training alone. On the other hand, Baumann et al. (2007) examined cortical areas that are concurrently activated during silent piano performance and motionless listening to piano sound, finding cross - modal activations in areas related to the absent modality that were higher in skilled musicians. Moreover, professional musicians show involuntary imagery of sound in pure motor tasks that resemble instrument performance and motor imagery in passive auditory tasks (Bangert et al., 2006). In an fMRI study by D'Ausilio et al. (2006), activation of motor-related areas in musicians occurred exclusively for rehearsed pieces, indicating that the prior training of auditory-motor interactions seems to be indispensable for cross-modal activations.

However, here we showed a reinforcement of motor performance by task-irrelevant auditory feedback, which did not serve as a cue for motor action and remained constant throughout the task. Some studies have indicated that there is a recruitment of motor areas when listening to untrained musical rhythms (Baumann et al., 2007; Chen et al., 2008) that might be involuntary due to the privileged link between auditory and motor areas (Zatorre et al., 2007). In our study, however, auditory stimuli were perceived as simultaneous to motor actions and not preceeding them, indicating that the auditory-motor interaction in musicians is more complex than previously thought.

Thus, our results indicate that the audio-motor integration capacities in musicians might go beyond their trained musical expertise such as instrument performance with relevant auditory outcome. More specifically, we provide novel evidence that 
task-irrelevant auditory feedback without any musical context, e.g., instruction to learn/perform a musical piece, is capable of reinforcing SRTT motor performance in musicians. An open yet important issue is whether or not a similar relationship exists also for non-musicians with a presumably different audio-motor network. Furthermore, the underlying neural mechanisms of taskirrelevant auditory feedback on motor performance in musicians and/or non-musicians still remain elusive and certainly require further investigation. However, in other domains it has been repeatedly shown that, e.g., speech and language functions are positively influenced by musical training which by itself is related to improved auditory information processing (for review, see Milovanov and Tervaniemi, 2011). This in turn could be one potential reason why musicians benefit from auditory feedback in the context of our SRTT. Alternatively, even though highly speculative and not explicitly tested in the present study, auditory information could also operate as an attentional enhancer increasing top-down control of motor performance.

In a similar SRTT study carried out in non-musicians, Stocker et al. (2003) did not find any effect of task-irrelevant auditory feedback on motor performance when the RBD was organized in a mixed way relative to a real piano distribution of tones. The authors hypothesized that auditory feedback has an effect on motor performance only when it is both contingent and compatible with motor responses. In our study, however, we were able to show that task-irrelevant auditory feedback has indeed an effect on motor performance in highly trained musicians even when the tone mapping of the RBD does not follow the same organization as a real piano. We believe that the main reason of these divergent results is that Stocker and colleagues investigated the effect of task-irrelevant auditory feedback in non-musicians only. It might be reasonable to assume that the generally enhanced motor skill learning capabilities in musicians when compared to non-musicians might overcome the effect of an incompatible RBD mapping.

A potential limitation of our study is that we did not control for explicit learning of the sequence even though most of the participants reported the feeling of a constant sequence at some point during task performance. In spite of that, the aim of the study was not to compare explicit vs. implicit learning, but to compare motor performance with or without auditory feedback where an explicit learning, if generally present, would not explain the differences between conditions. Nevertheless, we cannot exclude the possibility that differences between the VM and the VMA group at least partially depend on different contributions of explicit learning, which in turn might result in differences between groups. However, this effect would most likely result in

\section{REFERENCES}

Bangert, M., and Altenmuller, E. O. (2003). Mapping perception to action in piano practice: a longitudinal DC-EEG study. BMC Neurosci. 4, 26. doi:10.1186/1471-2202-4-26

Bangert, M., Haeusler, U., and Altenmuller, E. (2001). On practice: how the brain connects piano keys and piano sounds. Ann. N. Y. Acad. Sci. 930, 425-428.

Bangert, M., Peschel, T., Schlaug, G., Rotte, M., Drescher, D., Hinrichs, H., Heinze, H. J., and Altenmuller, E. (2006). Shared networks for auditory and motor processing in professional pianists: evidence from fMRI conjunction. Neuroimage 30, 917-926.

differences regarding SRTT learning (which we did not observe in our study). Furthermore, we were not able to identify significant differences in SRTT learning between musicians even though SRTT performance (RTs) was superior in the VMA as compared to the VM group. While the reason for this finding remains unclear at this point, it might be reasonable to assume that SRTT learning in the VMA group was restricted due to a ceiling effect. Additionally, we cannot exclude that other movement parameters such as peak velocity, force, movement onset, and/or kinematics might have improved during SRTT performance in both the VMA and VM group. Finally, faster RTs in the VMA group might be simply related to differences in motivation, and/or internalizing/learning the melody of the tones presented. However, differences in motivation as a sole mechanism seems to be unlikely since we did not observe any differences (pre vs. post) in fatigue or discomfort between groups. Furthermore, internalizing the melody of tones would potentially result in a better learning capability (which was not seen in our study sample) and/or in faster RTs through motor preparation, which could be considered an additional SRTT learning measurement that, however, was not acquired in the present study. Additionally, it is important to keep in mind that all subjects were required to keep the tempo of the GO signal and all key presses on the RBD before the GO signal were computed as errors. Since error rates are not different between groups, we are confident that anticipation cannot exclusively explain our research findings. However, a potential mechanism might be that the task-irrelevant auditory feedback helps keeping the tempo and therefore results in faster RTs and/or might allow being prepared for which key to press leading to overall faster RTs.

Further experiments addressing other space-pitch distributions with either existent or novel auditory-motor internal representations are needed in order to elucidate which role the space-pitch distribution has within the reported effects. Moreover, follow-up studies measuring different and finer components of motor performance are important to closely look at differences in learning when receiving task-irrelevant auditory feedback.

In summary, we have shown that musicians are significantly faster when performing a motor task with irrelevant auditory feedback not directly related with music performance as a form of near transfer (Hyde et al., 2009), exhibiting a role for mixed space-pitch auditory feedback in enhancing visually guided motor performance but not learning.

\section{SUPPLEMENTARY MATERIAL}

The Supplementary Material for this article can be found online at www.frontiersin.org/Auditory_Cognitive_Neuroscience/abstract/ 18682

Baumann, S., Koeneke, S., Schmidt, C. F., Meyer, M., Lutz, K., and Jancke, L. (2007). A network for audio-motor coordination in skilled pianists and nonmusicians. Brain Res. 1161 , 65-78.

Chen, J. L., Penhune, V. B., and Zatorre, R. J. (2008). Listening to musical rhythms recruits motor regions of the brain. Cereb. Cortex 18 2844-2854.

D'Ausilio, A., Altenmuller, E., Olivetti Belardinelli, M., and Lotze, M. (2006). Cross-modal plasticity of the motor cortex while listening to a rehearsed musical piece. Eur. J. Neurosci. 24, 955-958.

Hyde, K. L., Lerch, J., Norton, A., Forgeard, M., Winner, E., Evans, 
A. C., and Schlaug, G. (2009). Musical training shapes structural brain development. J. Neurosci. 29, 3019-3025.

Jancke, L., Schlaug, G., and Steinmetz, H. (1997). Hand skill asymmetry in professional musicians. Brain Cogn. 34, 424-432.

Lahav, A., Boulanger, A., Schlaug, G., and Saltzman, E. (2005). The power of listening: auditory-motor interactions in musical training. Ann. N. Y. Acad. Sci. 1060, 189-194.

Lim, V. K., Bradshaw, J. L., Nicholls, M. E., and Altenmuller, E. (2005). Enhanced P1-N1 auditory evoked potential in patients with musicians' cramp. Ann. N. Y. Acad. Sci. 1060, 349-359.

Milovanov, R., and Tervaniemi, M. (2011). The interplay between musical and linguistic aptitudes: a review. Front. Psychol. 2:321. doi:10.3389/fpsyg.2011.00321
Oldfield, R. C. (1971). The assessment and analysis of handedness: the Edinburgh inventory. Neuropsychologia 9, 97-113.

Pantev, C., Lappe, C., Herholz, S. C., and Trainor, L. (2009). Auditorysomatosensory integration and cortical plasticity in musical training. Ann. N. Y. Acad. Sci. 1169, 143-150.

Perez, M. A., Tanaka, S., Wise, S. P., Willingham, D. T., and Cohen, L. G. (2008). Time-specific contribution of the supplementary motor area to intermanual transfer of procedural knowledge. J. Neurosci. 28, 9664-9669.

Perez, M. A., Wise, S. P., Willingham, D. T., and Cohen, L. G. (2007). Neurophysiological mechanisms involved in transfer of procedural knowledge. J. Neurosci. 27, 1045-1053.
Schulz, M., Ross, B., and Pantev, C. (2003). Evidence for traininginduced crossmodal reorganization of cortical functions in trumpet players. Neuroreport 14 157-161.

Stocker, C., Sebald, A., and Hoffmann, J. (2003). The influence of response - effect compatibility in a serial reaction time task. Q. J. Exp. Psychol. A 56, 685-703.

Zatorre, R. J., Chen, J. L., and Penhune, V. B. (2007). When the brain plays music: auditory-motor interactions in music perception and production. Nat. Rev. Neurosci. 8, 547-558.

Conflict of Interest Statement: The authors declare that the research was conducted in the absence of any commercial or financial relationships that could be construed as a potential conflict of interest.

Received: 24 November 2011; accepted: 24 April 2012; published online: 16 May 2012.

Citation: Conde V, Altenmüller $E$, Villringer $A$ and Ragert $P$ (2012) Task-irrelevant auditory feedback facilitates motor performance in musicians. Front. Psychology 3:146. doi: 10.3389/fpsyg.2012.00146

This article was submitted to Frontiers in Auditory Cognitive Neuroscience, a specialty of Frontiers in Psychology. Copyright (c) 2012 Conde, Altenmüller, Villringer and Ragert. This is an openaccess article distributed under the terms of the Creative Commons Attribution Non Commercial License, which permits non-commercial use, distribution, and reproduction in other forums, provided the original authors and source are credited. 


\section{APPENDIX \\ STATISTICAL ANALYSES WITHOUT THE LEFT-HANDED PARTICIPANT}

In order to account for any potential noise that a left-handed participant could add in a right hand motor task, we decided to repeat the measurements of our dataset (SRTT performance (VM), comparison between SRTT VM, and VMA and SRTT learning) after excluding the data of the left-handed participant from the corresponding condition (VM).

\section{SRTT PERFORMANCE (VM)}

The repeated measures ANOVA revealed a significant effect of factor TIME, indicating a significant decrease in RTs along TS trials (from TS1 to TS28) in the VM condition (ANOVARM with factor TIME: $F(27,270)=15.761 ; p<0.0001)$.

\section{COMPARISON BETWEEN SRTT VM AND VMA}

When comparing performance (measured as differences in RTs along trials of the TS - from TS1 to TS28), a significant difference between conditions could be found [ANOVARM with factor TIME x CONDITION: $F(27,270)=1.658 ; p<0.024]$, indicating that there was a differential decrease in RTs along time depending on condition.

\section{SRTT LEARNING}

No differences in learning could be addressed either when taking into account both differences between first and last TS and US trials [(TS1-TS28)/(US(first)-US(last))] (percentage of learning: $35.38 \pm 9.39 \%$ in VM condition; $25.03 \pm 5.05 \%$ in VMA condition; independent samples $t$-test; $p=0.332$ ); and the last US-TS difference only [difference in RT (ms): $122.00 \pm 22.00 \mathrm{~ms}$ in VM condition; $72.16 \pm 11.34 \mathrm{~ms}$ in VMA condition; independent samples $t$-test; $p=0.051$, non-significant trend].

These results indicate that the left-handed participant did not substantially change the results or add noise to the dataset, thus validating its inclusion. 